\title{
Power Optimized Programmable Embedded CONTROLLER
}

\author{
M.Kamaraju $^{1} \quad$ Dr.K.Lal Kishore ${ }^{2} \quad$ Dr.A.V.N.Tilak ${ }^{3}$ \\ ${ }^{1}$ Dept. of ECE, Gudlavalleru Engineering College, Gudlavalleru - India \\ madduraju@yahoo.com \\ ${ }^{2}$ Director,SIT, Jawarlal Nehru Technological University ,Hyderabad- India \\ lalkishorek@yahoo.com \\ ${ }^{3}$ Dept. of ECE,Gudlavalleru Engineering College, Gudlavalleru - India \\ avntilak@yahoo.com
}

\begin{abstract}
Now a days, power has become a primary consideration in hardware design, and is critical in computer systems especially for portable devices with high performance and more functionality. Clock-gating is the most common technique used for reducing processor's power. In this work clock gating technique is applied to optimize the power of fully programmable Embedded Controller (PEC) employing RISC architecture. The CPU designed supports i) smart instruction set, ii) I/O port, UART iii) on-chip clocking to provide a range of frequencies, iv) RISC as well as controller concepts. The whole design is captured using VHDL and is implemented on FPGA chip using Xilinx. The architecture and clock gating technique together is found to reduce the power consumption by $33.33 \%$ of total power consumed by this chip.
\end{abstract}

\section{KEYWORDS}

Power, Clock Gating, RISC, FPGA, Xilinx

\section{INTRODUCTION}

Low power consumption in embedded systems [1] has become a key factor for many applications. Portable applications, needing long battery life together with high peak- performance, are demanding a very careful design at all levels.

The most important factor contributing to the energy consumption is the switching activity [2] [3] .Once the technology and supply voltage have been set, major energy savings come from the careful minimization of switching activity.

While some switching activity is functional, i.e. it is required to propagate and manipulate information; there is a substantial amount of unnecessary activity in almost all digital circuits. Unnecessary switching activity arises from spurious transitions [4] due to unequal propagation delays (glitches) and transitions occurring within units that are not participating in a computation. One way to avoid these activities is by dynamically turning off the clock[5] to unused logic or peripherals .

Existing microprocessors [6], especially in the category of microcontrollers, often have the capability of partially gating the clock signal when they fetch and execute NOP or other minimal-activity instructions and also when a peripheral is powered-down. This does not totally eliminate spurious power consumption. Other processors [7] can enter low-power operating modes, disabling the clock signal generator (Xtal oscillator and PLL). However, this is not 
totally efficient in important applications. In [8] optimization of power is achieved by taking assistance of compiler while executing instructions. However hardware approach proposed in this work reduces the power without compromising speed performance of the chip.

In the proposed PEC, the control unit is designed to have the capability of gating the clock signal when they fetch and execute instructions. On-chip clocking [9] mechanism is employed to synchronize with on-chip peripherals/memory and with the external bus. For power optimization of integrated circuits it is relevant to understand the causes of power dissipation. Clock power[10] dominates the total power consumed by a microcontroller as the clock is fed to most of the circuit blocks. Charge/discharge power given by $\mathrm{P}=\mathrm{f} \mathrm{C}_{\mathrm{L}} \mathrm{V}_{\mathrm{dd}} \mathrm{V}_{\mathrm{s}}$, dominates the total power dissipation of the chip. The frequency $f$ of the clock cannot be reduced as it effects the speed of the chip. When output swings from 0 to $V_{d d}$ then $P$ varies as square $V_{d d}$ .However lowering $\mathrm{P}$ by reducing power supply voltage to $2 \mathrm{~V}$ or less is found to lead to several problems[11] [12] like decrease in drivability of MOSFET and increase in gate delay time.

\section{Programmable Embedded Controller Architecture}

Architecture of PEC is shown in Figure.1. Various blocks in the architecture are register file, ALU, RAM, ROM, UART, I/O Ports, BCD to 7 segment driver, Control unit, and clocker, designed to perform particular task. Register File is a set of registers that are modeled as RAM of 16 bit words, used to store intermediate values during instruction processing. The ALU performs 16 bit operations. The Read Only Memory (ROM) is 256 bytes with 16 bit word length and is used to store the instruction data. The Random Access Memory (RAM) $1 \mathrm{~K} \times 16$ is used to store temporary data. Port 0 and Port 1 are two ports which are configured as output and input ports respectively.

A display driver for BCD to 7 segment display is designed to drive the 7 segment display unit. The control unit generates various control signals to all other blocks to execute desired task specified by the instructions. The PEC is initiated by the reset signal whenever reset signal asserts high, the controller generate appropriate signals to load the PC address of the ROM. The external interrupt mechanism activates on any hardware interrupt or reset signal arriving at the controller when it is in idle mode.

\subsection{On-Chip Clocking Mechanism}

The frequency of the application specific hardwired oscillator shown in Figure.2 is programmable by means of the 4-bit number (control word value) contained in the dedicated register r_osc. On-chip clocking is used to obtain different frequencies ranging from $44 \mathrm{MHz}$ to $134 \mathrm{MHz}$ by changing the control word values as shown in Figure. 3. 


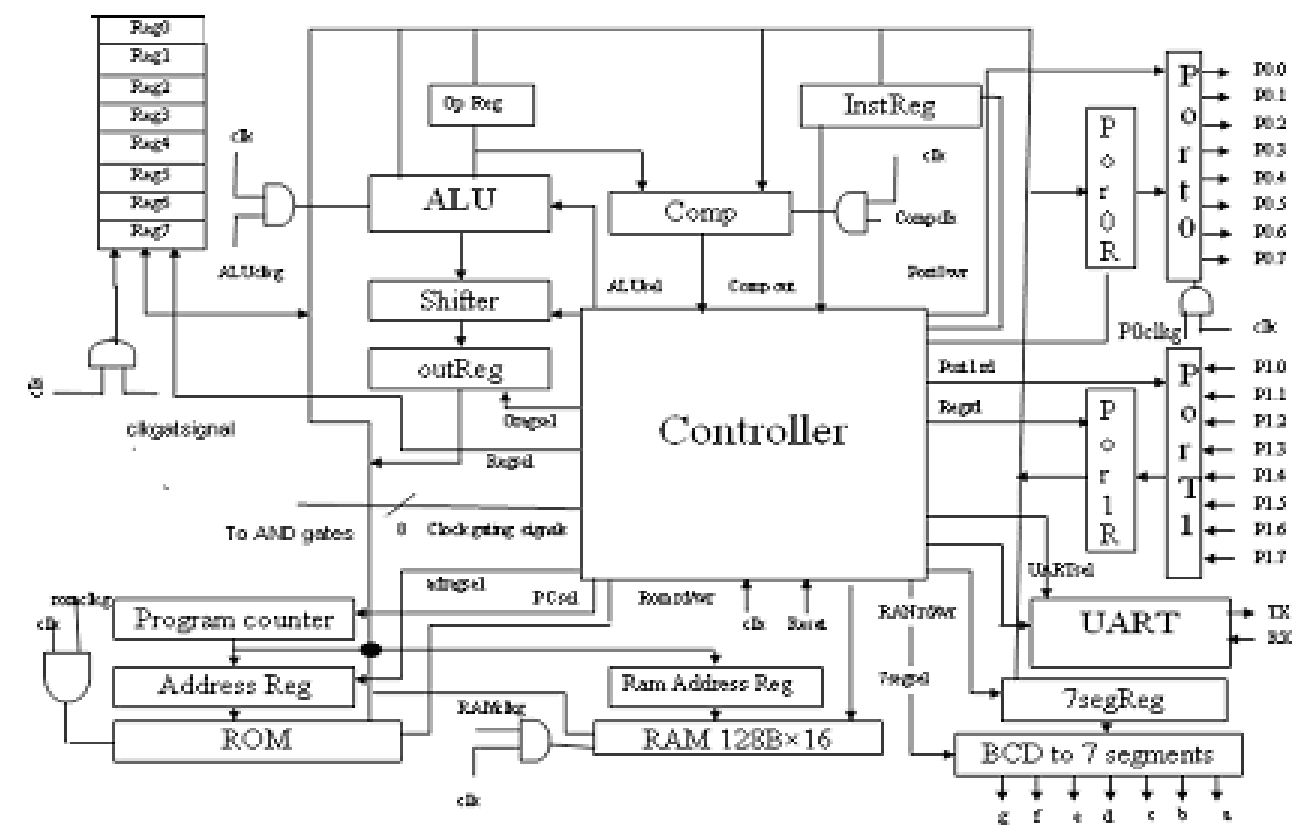

Clock

Clocker

Figure.1 Architecture of Programmable Embedded Controller

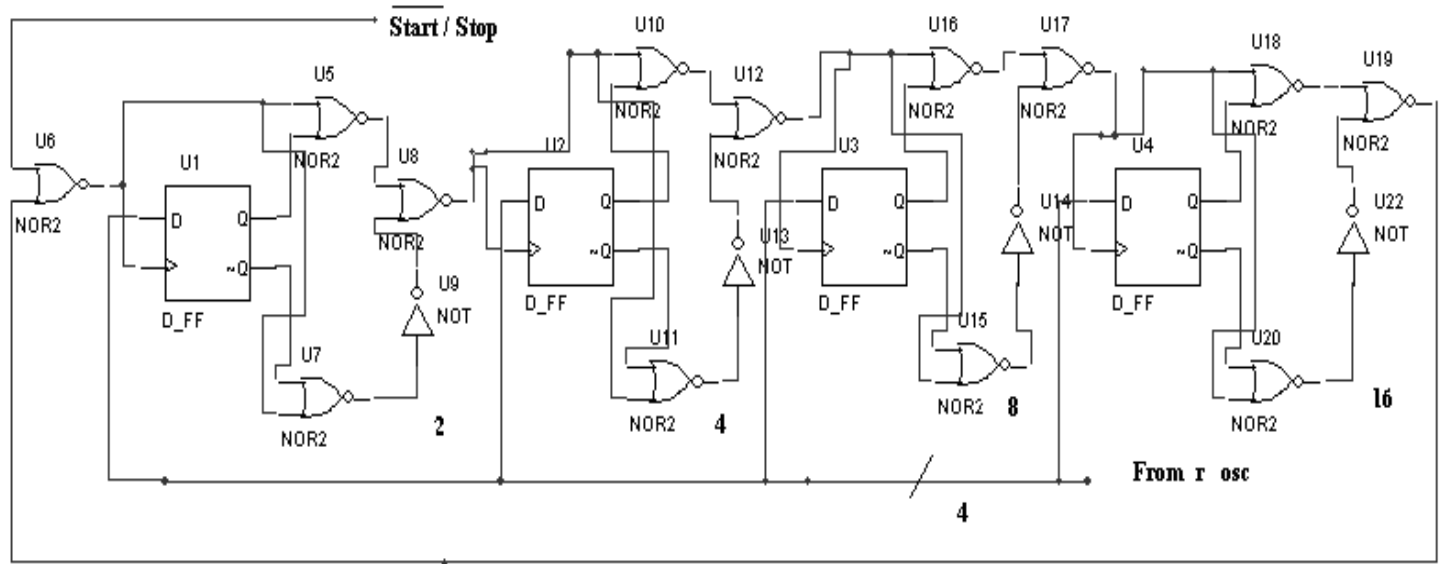

CLK

Figure.2 Oscillator Circuit 


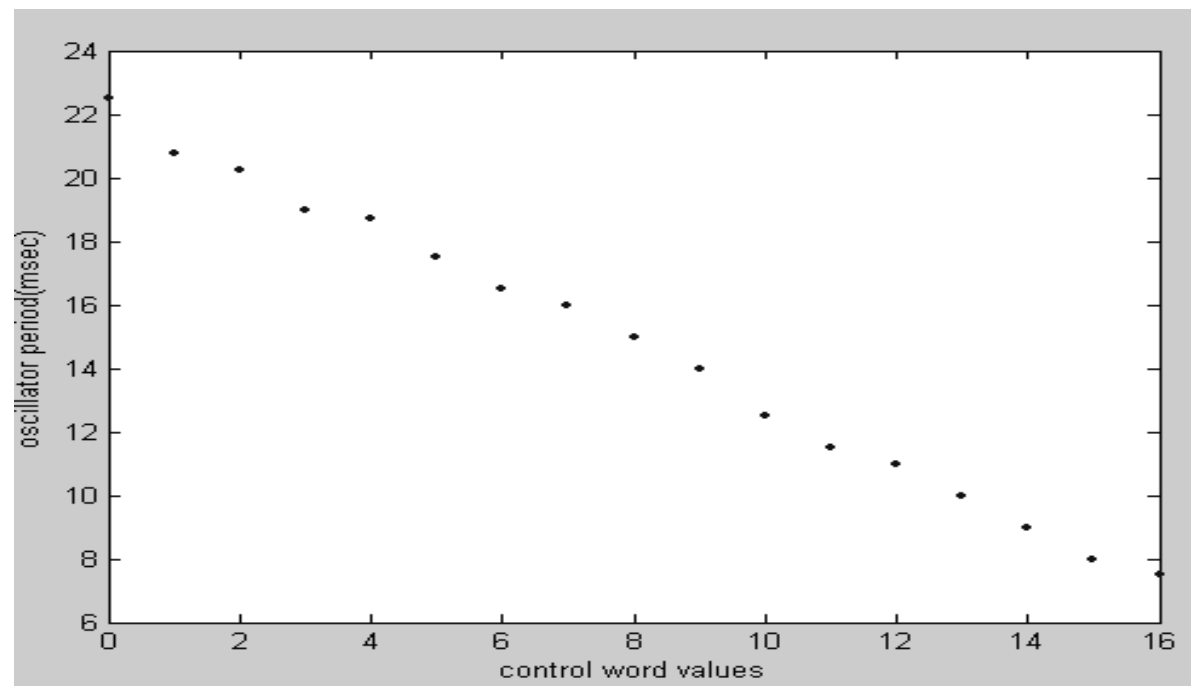

Figure.3 Oscillator Cycle Time vs. Control Word Value

\subsection{Clock Gating}

Figure. 4(a) shows the schematic of latch element. A significant amount of power is consumed during charge/discharge cycle of the cumulative gate capacitance $\mathrm{C}_{\mathrm{g}}$ of the latch, when the clock is fed directly and there is no change in the clock cycle[13]. Figure. 4(b) shows the latch with gated clock. By gating the clock [14] [15] , charge/discharge of $C_{g}$ can be effected only when there is change in the clock cycle thus saving power.

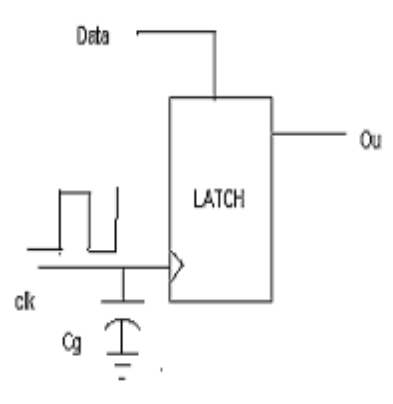

(a)

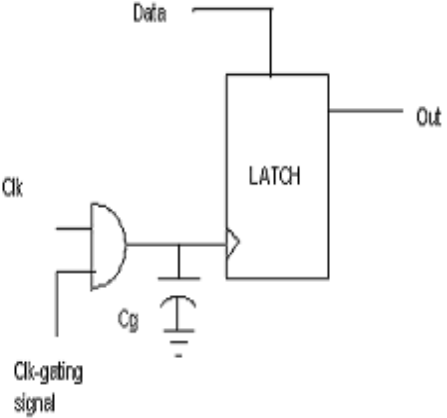

(b)

Figure.4 Schematic of Latch Element (a) without clock gating (b) with clock gating

The controller shown in Figure. 1 supports predefined smart instruction set having length of 16 bits each. Gated clock signal generated by the control unit allows the clock to be fed only to the active blocks and not to the unused blocks.

\subsection{Control Unit}

The control unit provides all of control signals to regulate the data traffic and necessary signals to perform the desired functions. The control unit architecture contain a state machine that 
causes all appropriate signal values to update based on current state and input signals and produce a next state for state machine. The control unit performs two processes. The first is a combinational process (not clocked) that examines the current state and all inputs and produces output control signals and next state output. The second is the sequential process (having a clock) that is used to store the current state and copy of the next state to the current state.

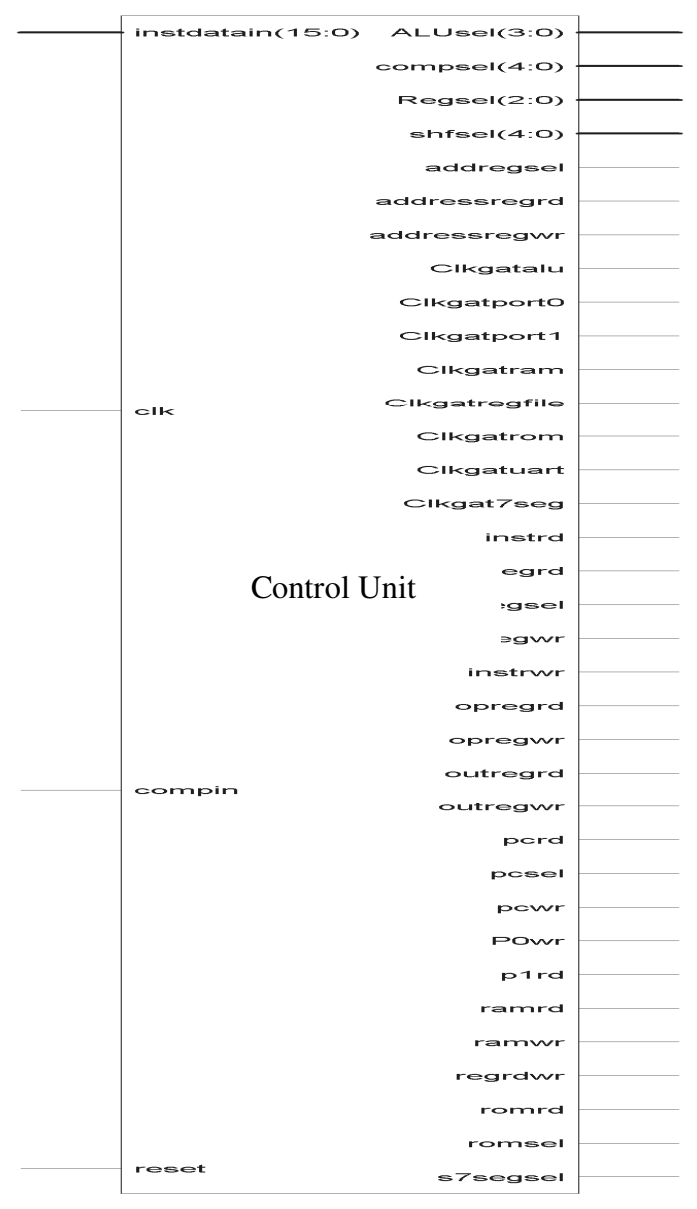

Figure.5 Signals of Control Unit

If the reset signal is high the sequential process set the current state value to reset1, the first state of the reset sequence. The logic for clock gating is implemented within the control unit. The controller generate appropriate clock gating signal to reduce power consumption of the chip. When the control unit decodes the opcode of the instruction, the control unit generates control signals as shown in Figure.5, to execute the instruction.

By implementing the instructions given in the instruction set (Table 1) does not cause any functional limitation, but enables an effective way of power saving through generation of gated clock signals. All the instructions are of length 2 bytes and of direct addressing mode type. Instruction set includes Load, Store, Branch, ALU and Shift instructions. 
Table 1 Instruction Set

\begin{tabular}{|c|c|c|}
\hline OPCODE & INSTRUCTION & DESCRIPTION \\
\hline 00000 & NOP & No operation \\
\hline 00001 & LOAD & Load register \\
\hline 00010 & STORE & Store the register \\
\hline 00011 & MOVE & Move the value into the register \\
\hline 00100 & LOADI & Load the register with immediate value \\
\hline 00101 & $\mathrm{BI}$ & Branch to immediate address \\
\hline 00110 & BGTI & Branch greater than to immediate address \\
\hline 00111 & INC & Increment \\
\hline 01000 & DEC & Decrement \\
\hline 01001 & AND & Logical AND two registers \\
\hline 01010 & OR & Logical OR two register \\
\hline 01011 & XOR & Logical XOR two register \\
\hline 01100 & NOT & Logical NOT the register \\
\hline 01101 & ADD & Add two registers \\
\hline 01110 & SUB & Subtract two registers \\
\hline 01111 & ZERO & Zero a register \\
\hline 10000 & PORT0 & Port 0 write \\
\hline 10001 & BLT & Branch lass than \\
\hline 10010 & BNEQ & Branch not equal \\
\hline 10011 & PORT1 & Port 1 read \\
\hline 10100 & BGT & Branch greater than \\
\hline 10110 & $\mathrm{BCH}$ & Branch all the time \\
\hline 10111 & BEQ & Branch if equal \\
\hline 11000 & B7S & 7 segment driver \\
\hline 11001 & BLTE & Branch less than or equal \\
\hline 11010 & SHL & Shift left \\
\hline 11011 & SHR & Shift right \\
\hline 11100 & ROR & Rotate right \\
\hline 11101 & ROL & Rotate lrft \\
\hline 11110 & UARTS & UART sel. \\
\hline
\end{tabular}

\section{RESULTS}

The entire design is captured in VHDL and simulated using Xilinx tool. The simulation results of control unit are presented in Figure.6. 


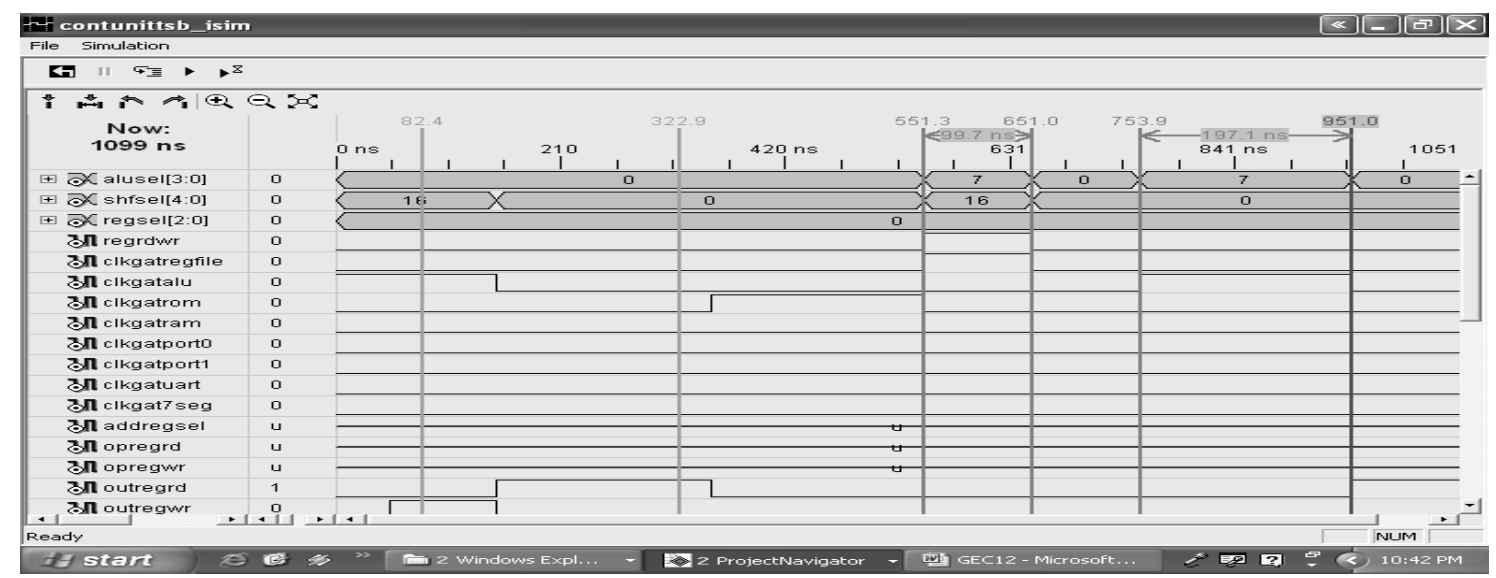

Figure.6 Control Unit Simulation Results

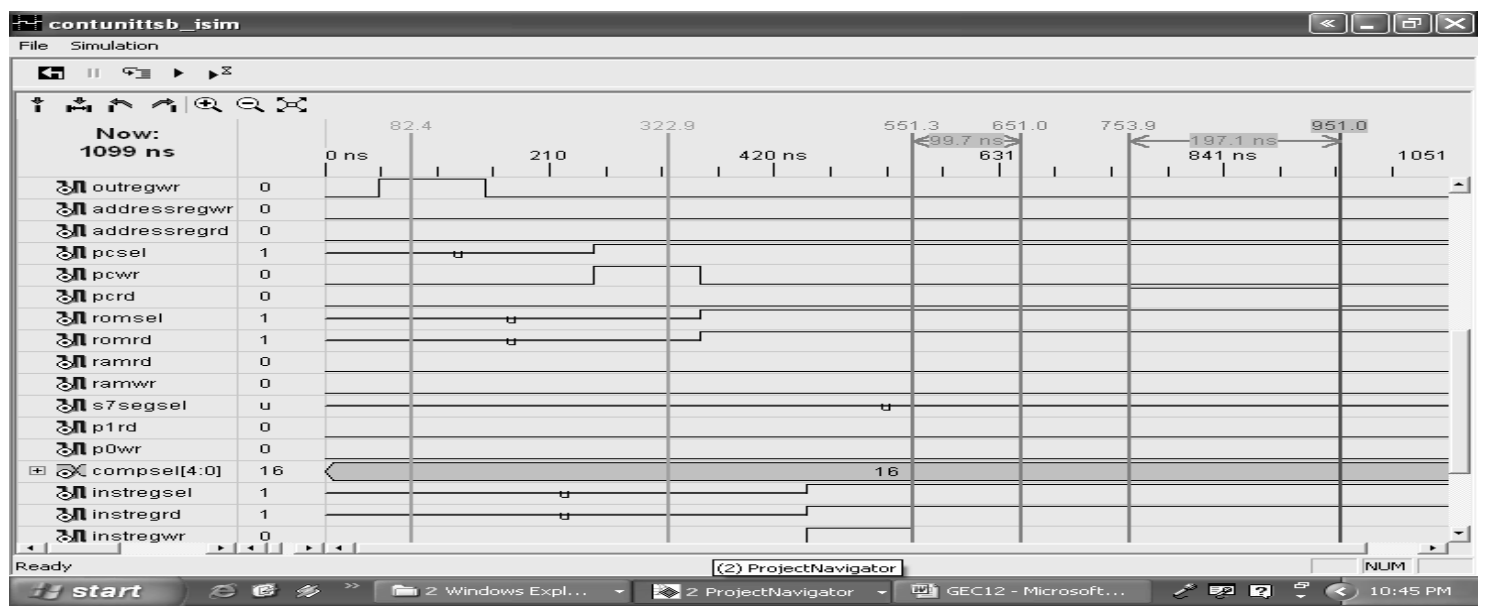

Figure.6 Control Unit Simulation Results (contd...)

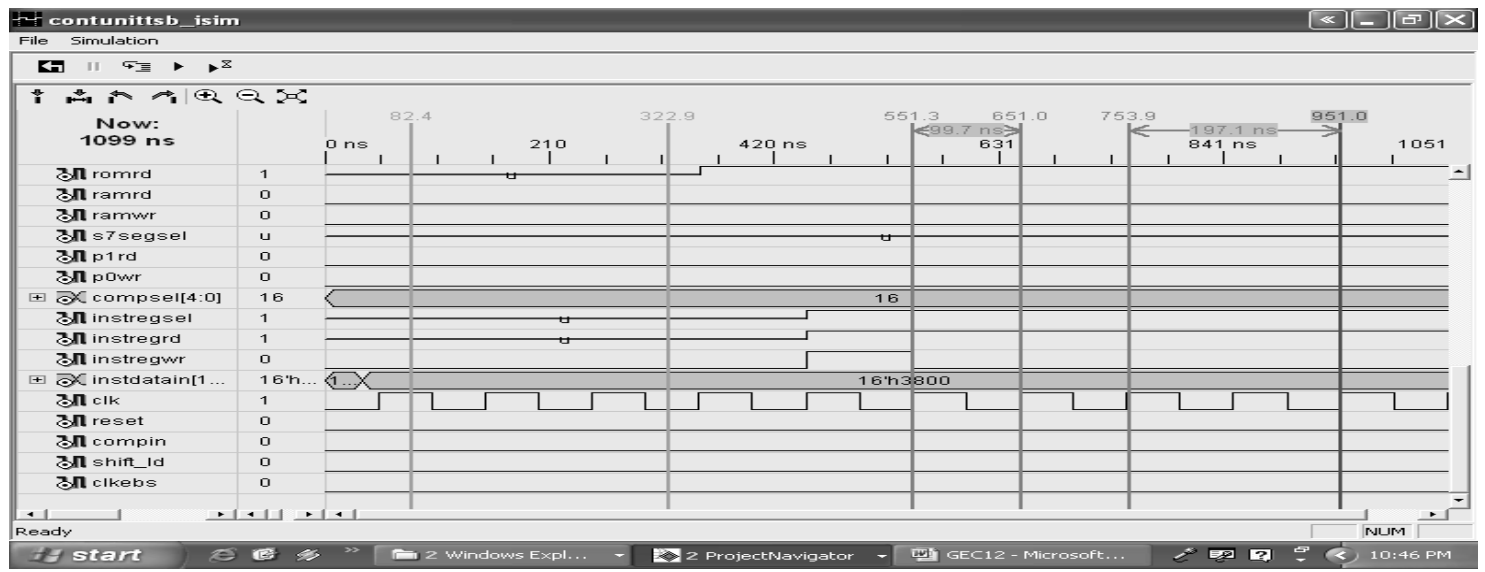

Figure.6 Control Unit Simulation Results (contd...) 


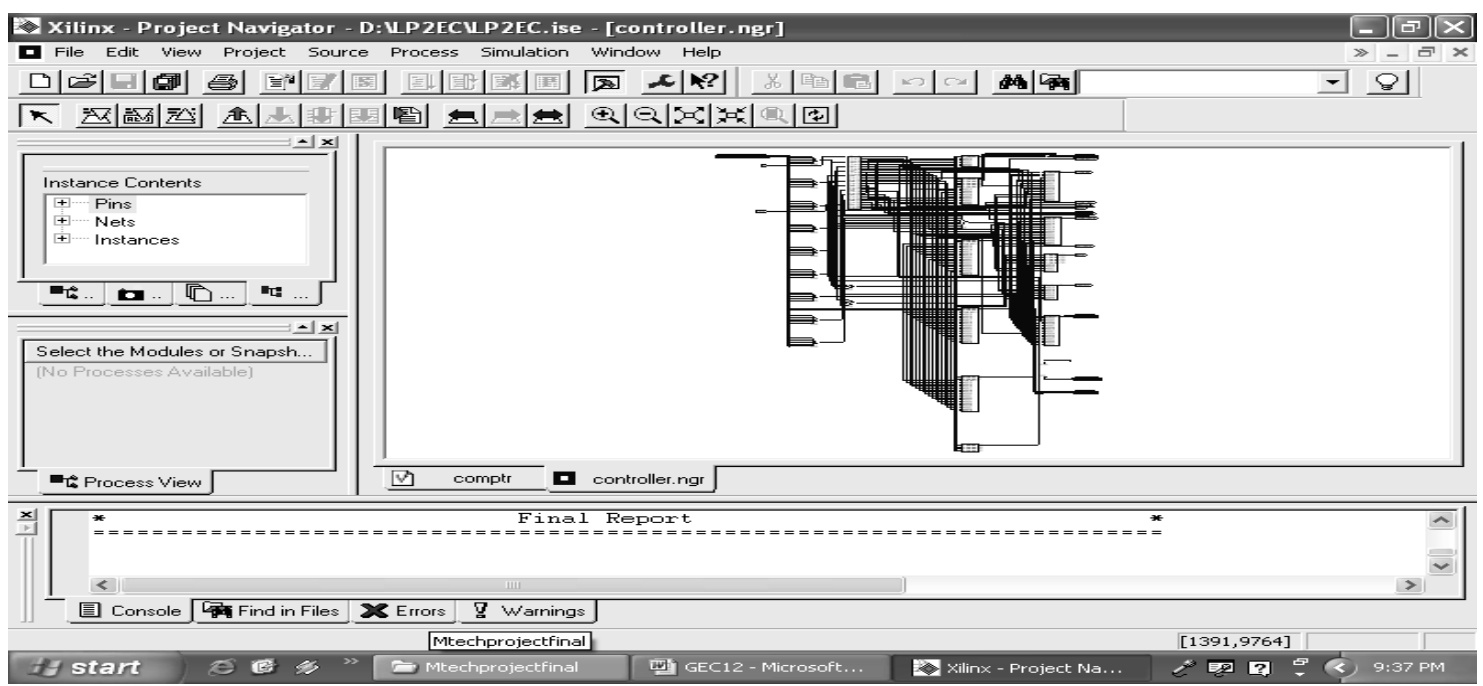

Figure.7 Control Unit RTL Schematic

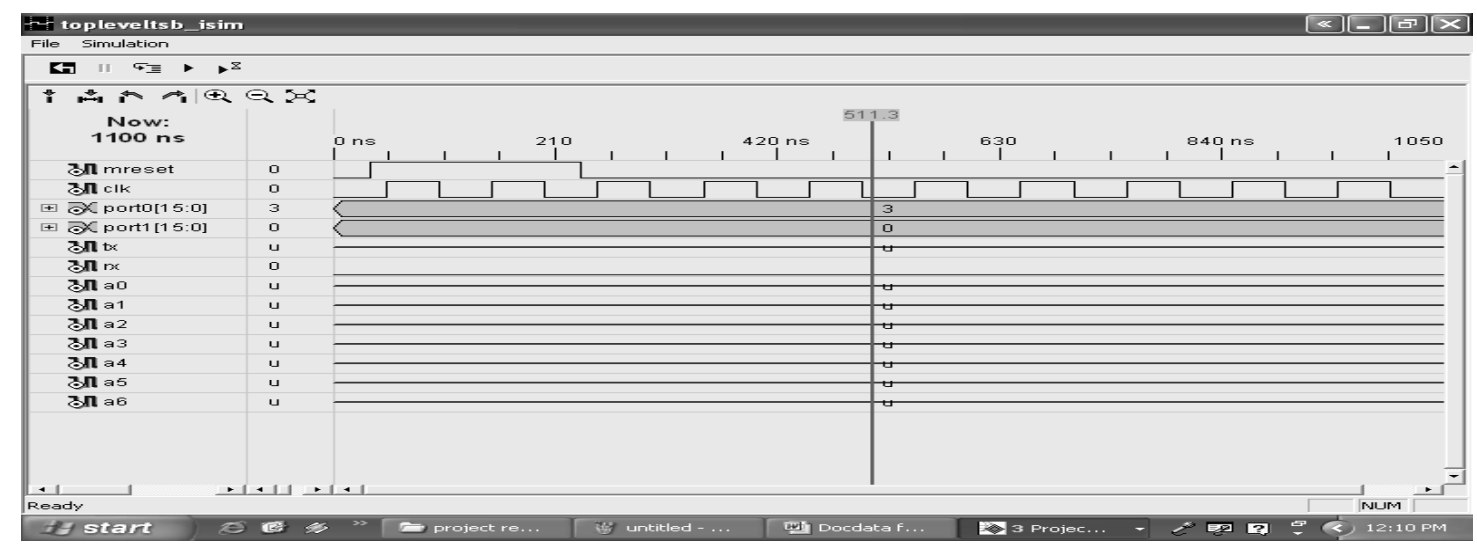

Figure. 8 Top Level Module Simulation Results

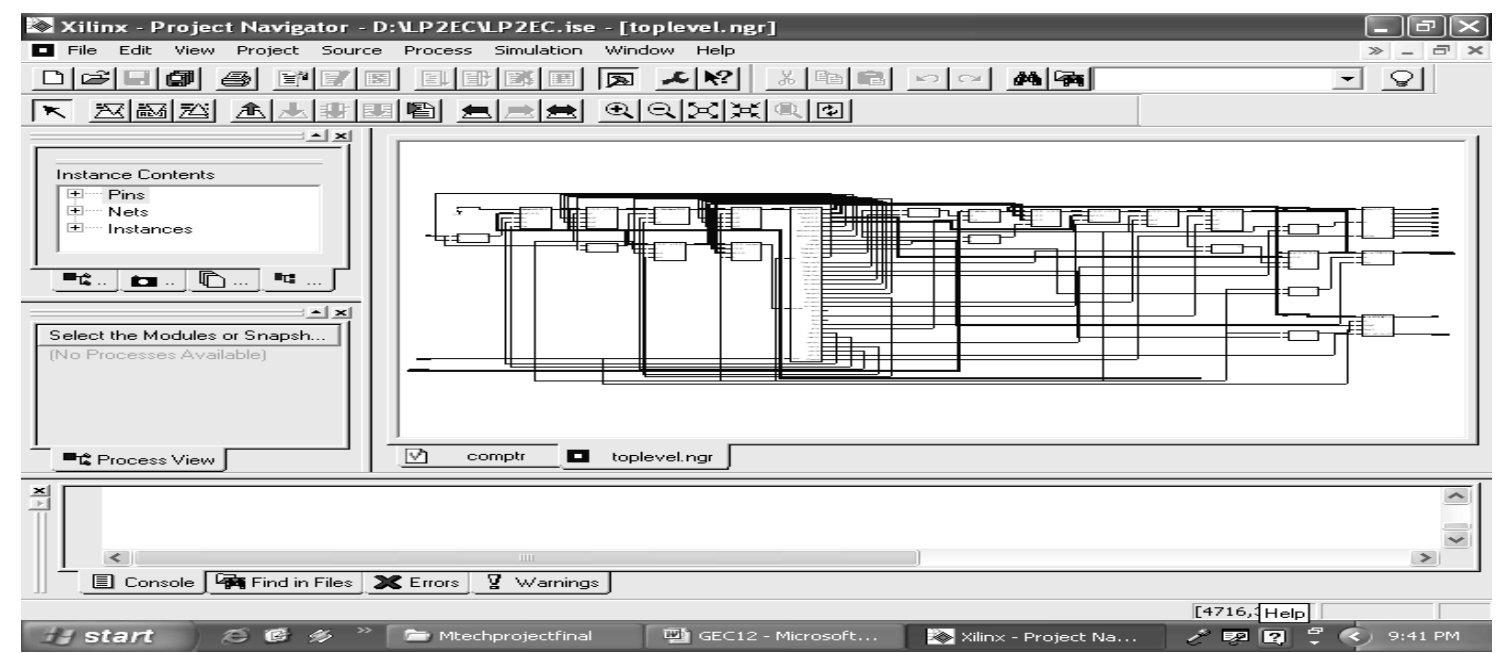

Figure. 9 Top Level Module RTL Schematic 


\subsection{Power Analysis}

The estimation of power consumption of each module is done using Xilinx Xpower's tool. The graphical representation of power consumed in various modules is shown in Figure.10.

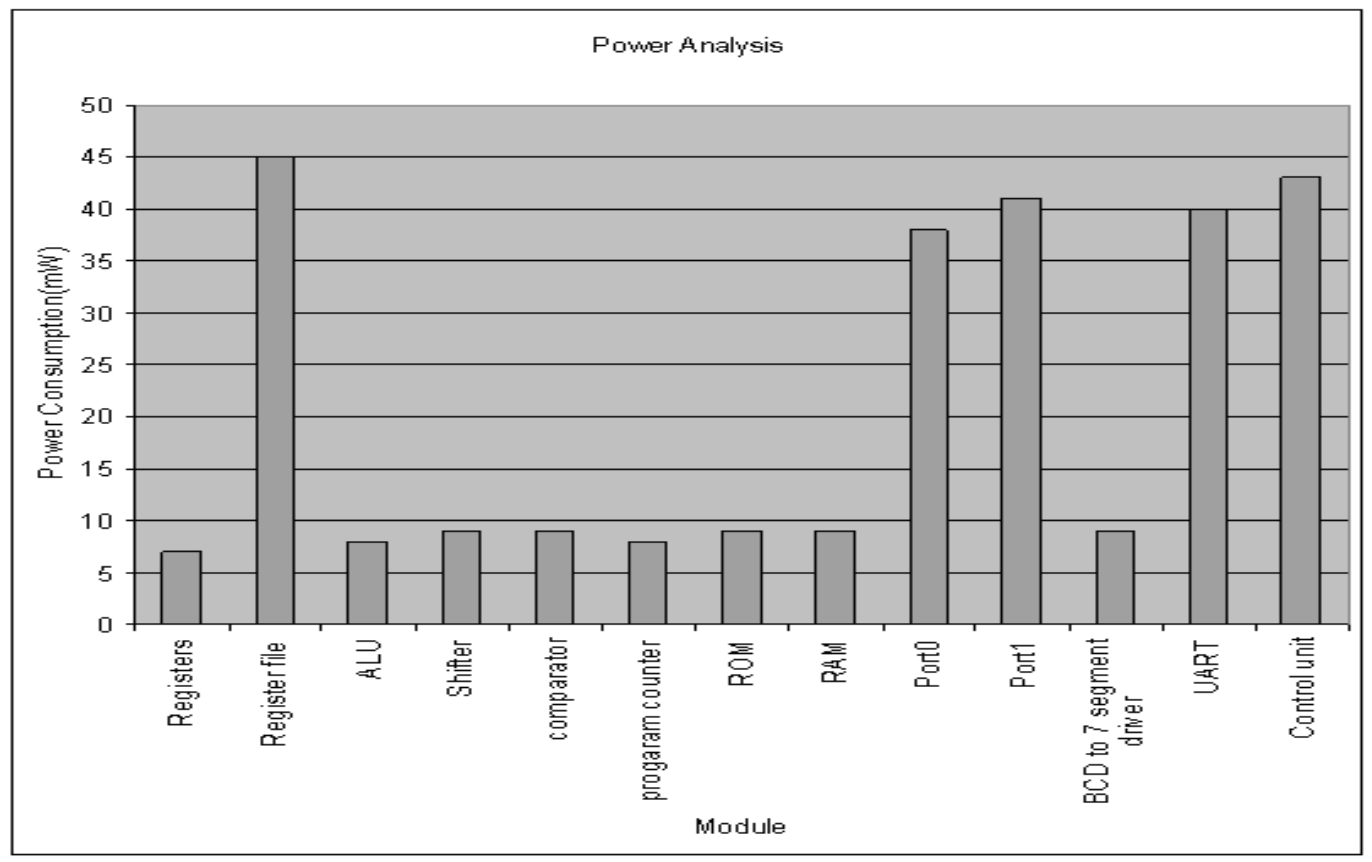

Figure.10 Power Analysis

Total power consumption is estimated to be $273 \mathrm{~mW}$ without clock gating and only $182 \mathrm{~mW}$ after clock gating technique is employed, thus achieving a power saving of 33.33\% (Figure.11).

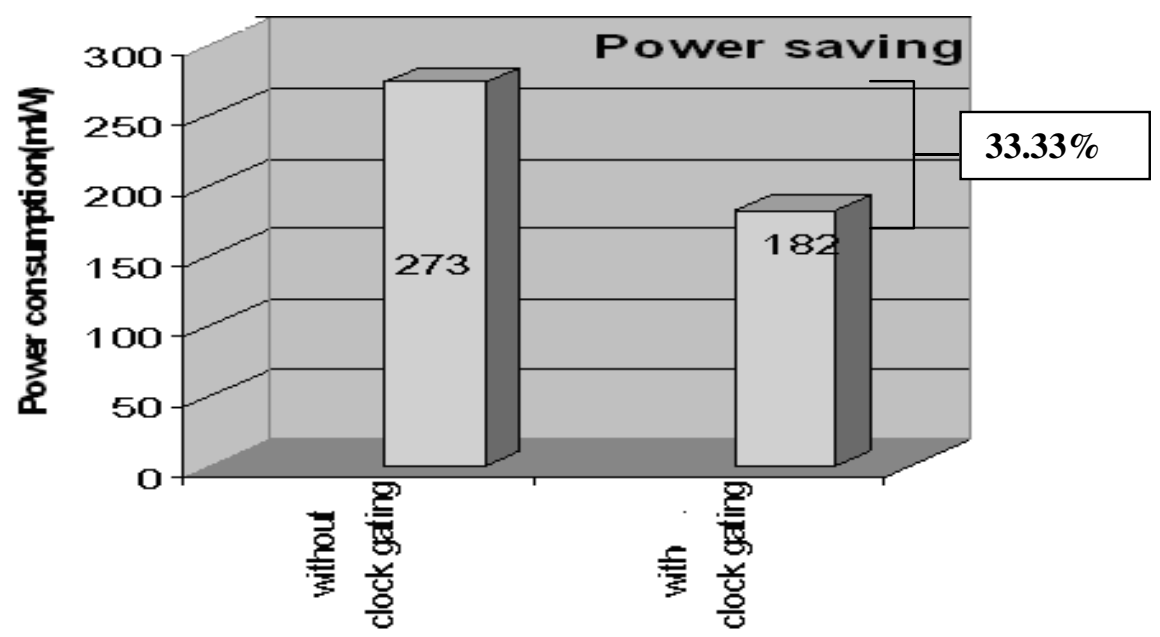

Figure.11 Comparison of Power Consumed without and with Clock Gating 


\subsection{Characteristics of the Chip}

The characteristics of the designed chip are

$\begin{array}{lc}\text { Architecture } & \text { RISC } \\ \text { Optimization } & \text { Power } \\ \text { Instructions } & 2 \text { byte } \\ \text { ROM } & 256 \text { bytes } \\ \text { RAM } & 1 \mathrm{~KB} \\ \text { ALU } & 16 \mathrm{bit} \\ \text { Power Supply } & 2.4 \mathrm{~V} \\ \text { Power Dissipation } & 3.62 \mathrm{~mW} / \mathrm{MHz}\end{array}$

\section{Conclusions}

The need for low power systems is being driven by many market segments. There are several approaches to reducing the power. In this work clock gating technique is applied to optimize the power of fully programmable embedded controller employing RISC architecture. The whole design is captured using VHDL language and is implemented on FPGA chip using Xilinx .The chip has less hardware complexity as this works based on single addressing mode to access the data for processing. The architecture and clock gating technique together have reduced the power consumption by $33.33 \%$ of total power consumed by the chip. This clock gating technique can be applied from chip level to module and then eventually to systems.

\section{REFERENCES}

[1] Haris Lekatsas, Jorg Henkel, Wayne Wolf, "A Decomposed Architecture of Low Power Embedded Systems ," Proc. of the 2000 IEEE International Conference on Computer Design: VLSI in Computer and Processors.

[2] Qing Wu, Massoud Pedram, and Xunwei Wu "Clock-Gating and Its Application to Low Power Design of Sequential Circuits" IEEE Transactions on Circuits and Systems-1: Fundamental Theory and Applications, Vol.47, No.103, March 2000, pp .415-420.

[3] Michael K.Gowan, Larry L.Biro, Daniel B.Jackson "Power Considerations in the Design of the Alpha 21264 Microprocessor, Transaction ID: 1998 ACM 0-89791-964-5/98/06, pp .726-731

[4] Jatuchai Pangjun and Sachin S.Saptnekar "Low-Power Clock Distribution Using Multiple Voltages and Reduced Swings" IEEE Transactions on Very Large Scale Integration(VLSI) Systems, Vol.10, No.3, June 2002, pp .309-318.

[5] David Brooks, Vivek Tiwari, Margaret Martonosi "Wattch:A Framework for ArchitecturalLevel Power Analysis and Optimizations” Transaction ID:ACM 2000 1-58113-232-8/00/6, pp .83-94.

[6] Rolf Hakenes, Yiannos Manoli, “A Novel Low - Power Micoprcessor Architecture” Trans. Id: 0-7695-0801-4/00\$10.00 2000 IEEE, pp.141- 146.

[7] Bill Moyer, "Low Power Design for Embedded Processor" Proc. of IEEE, Vol.89, No.11, November 2001.

[8] Divya Arora, Srivaths Ravi,Anand Raghunathan,Niraj K.Jha, "Hardware - Assisted Run - Time Monitoring for secure Program Execution on Embedded processors" Proc. IEEE, Vol.14,No.12,December 2006, pp 1295 - 1307. 
[9] Srikanth Rao and S.K. Nandy, "Power Minimization using Control Generated Clock", DAC 2000, Los Angeles, California.

[10] Sulaiman, D.R., "Using Clock Gating Technique for Energy Reduction in Portable Computers", International Conference on Computer and Communication Engineering, ICCCE 2008, 13-15 May 2008, pp.839 - 842 .

[11] Srinivas Devadas, Sharad Malik.A , "Survey of Optimization Techniques Targeting Low Power VLSI circuits", 32 ${ }^{\text {nd }}$ ACM/IEEE Design Automation Conference.

[12] Brock Barton, Massoud Pedram "Low Power Electronics and Design" IEEE Transactions on Very Large Scale Integration (VLSI) Systems, Vol.5, No.4, December 1997, pp .349-350.

[13] David E.Duarte, N.Vijaykrishnan, and Mary Jane Irwin “A Clock Power Model to Evaluate Impact of Architectural and Technology Optimizations" IEEE Transactions on Very Large Scale Integration (VLSI) Systems, Vol.10, No.6, December 2002, pp .844-855.

[14] Monica Donno, Alessandro Ivaldi, Luca Benini, Enrico Macii "Clock-Tree Power Optimization based on RTL Clock-Gating", DAC 2003, pp .622-627.

[15] W.G.Osborne, W.Luk, J.G.F.Coutinho and O.Mencer "Reconfigurable Design with Clock Gating” IEEE Transaction 978-1-4244-1985-2/08, 2008, pp .187-194.

\section{Authors}

Dr.K.Lal Kishore, obtained Master's degree and Ph.D. from Indian Institute of Science (IISC) Bangalore.He had published more than 76 research papers in International/Natioanl journals and presented papers in International / National Conferences.He is best teacher awardee from the Government of Andhrapardesh,S.V.C.Aiya Memorial Award from IETE,Merit Award from DEC, Ethopia, Bapu Seetharam Memorial Award from IETE and has many other academic distinctions to his credit.He wrote books on Electronic Devices, Circuit Analysis,Linear IC Applications,Electronic Measurements and Instrumentation and VLSI Design.

Dr.A.V.N.Tilak, obtained Master's degree from Indian Institute of Technology, Kanpur and Ph.D. from Indian Institute of Technology, Madras during 1984 and 1997 respectively. He is a Fellow of Institution of Electronics And Telecommunication Engineers, India and Life Member of Indian Society for Technical Education.

M.Kamaraju, obtained his Bachelor's degree \& Master's degree from Andhra University. Areas of interest are Microprocessors, Microcontrollers, Embedded Systems, Low Power VLSI. He is a fellow of Institution of Electronics and Telecommunication Engineers, India \& Member of VSI.
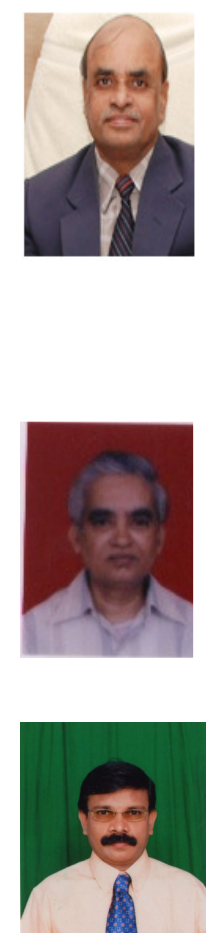